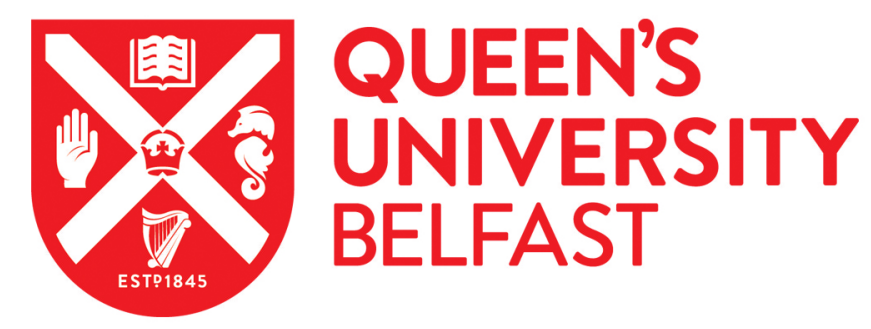

\title{
Dynamics of electric fields driving the laser acceleration of multi-MeV protons
}

Romagnani, L., Fuchs, J., Borghesi, M., Antici, P., Audebert, P., Ceccherini, F., Cowan, T., Grismayer, T., Kar, S., Macchi, A., Mora, P., Pretzler, G., Schiavi, A., Toncian, T., \& Willi, O. (2005). Dynamics of electric fields driving the laser acceleration of multi-MeV protons. Physical Review Letters, 95(19), 195001-195001-4. [195001]. https://doi.org/10.1103/PhysRevLett.95.195001

Published in:

Physical Review Letters

Document Version:

Publisher's PDF, also known as Version of record

Queen's University Belfast - Research Portal:

Link to publication record in Queen's University Belfast Research Portal

\section{General rights}

Copyright for the publications made accessible via the Queen's University Belfast Research Portal is retained by the author(s) and / or other copyright owners and it is a condition of accessing these publications that users recognise and abide by the legal requirements associated with these rights.

Take down policy

The Research Portal is Queen's institutional repository that provides access to Queen's research output. Every effort has been made to ensure that content in the Research Portal does not infringe any person's rights, or applicable UK laws. If you discover content in the Research Portal that you believe breaches copyright or violates any law, please contact openaccess@qub.ac.uk. 


\title{
Dynamics of Electric Fields Driving the Laser Acceleration of Multi-MeV Protons
}

\author{
L. Romagnani, ${ }^{1}$ J. Fuchs, ${ }^{2,3}$ M. Borghesi, ${ }^{1, *}$ P. Antici, ${ }^{2,4}$ P. Audebert, ${ }^{2}$ F. Ceccherini, ${ }^{5}$ T. Cowan, ${ }^{3}$ T. Grismayer, ${ }^{6}$ S. Kar, ${ }^{1}$ \\ A. Macchi, ${ }^{5}$ P. Mora, ${ }^{6}$ G. Pretzler, ${ }^{7}$ A. Schiavi, ${ }^{4}$ T. Toncian, ${ }^{7}$ and O. Willi ${ }^{7}$ \\ ${ }^{1}$ School of Mathematics and Physics, The Queen's University of Belfast, Belfast, Northern Ireland, United Kingdom \\ ${ }^{2}$ Laboratoire pour l'Utilisation des Lasers Intenses (LULI), UMR 7605 CNRS-CEA-École Polytechnique-Univ, \\ Paris VI, 91128 Palaiseau, France \\ ${ }^{3}$ Physics Department, MS-220, University of Nevada, Reno, Nevada 89557, USA \\ ${ }^{4}$ Dipartimento di Energetica, Università di Roma 1 "La Sapienza," Roma, Italy \\ ${ }^{5}$ Istituto Nazionale per la Fisica della Materia (INFM), Dipartimento di Fisica “E. Fermi," Università di Pisa, Pisa, Italy \\ ${ }^{6}$ Centre de Physique Théorique, UMR 7644 CNRS-École Polytechnique, 91128 Palaiseau, France \\ ${ }^{7}$ Institut für Laser-und Plasmaphysik, Heinrich-Heine-Universität, Düsseldorf, Germany
}

(Received 16 May 2005; published 31 October 2005)

\begin{abstract}
The acceleration of multi-MeV protons from the rear surface of thin solid foils irradiated by an intense $\left(\sim 10^{18} \mathrm{~W} / \mathrm{cm}^{2}\right)$ and short $(\sim 1.5 \mathrm{ps})$ laser pulse has been investigated using transverse proton probing. The structure of the electric field driving the expansion of the proton beam has been resolved with high spatial and temporal resolution. The main features of the experimental observations, namely, an initial intense sheath field and a late time field peaking at the beam front, are consistent with the results from particle-in-cell and fluid simulations of thin plasma expansion into a vacuum.
\end{abstract}

PACS numbers: 52.38.Kd, 52.65.-y, 52.70.Ds

The acceleration of multi-MeV ion beams following the interaction of short $(\tau \lesssim 1 \mathrm{ps})$ and intense $(I>$ $10^{18} \mathrm{~W} / \mathrm{cm}^{2}$ ) laser pulses with thin solid foils has been one of the most active areas of research in high field science in the last few years [1-3]. The high brightness, multi-MeV energy spectral cutoff, and the excellent degree of collimation and laminarity [4,5] distinguish these ion beams from those observed in earlier works [6], making them suitable for a range of applications [7-11].

In the laser intensity regime considered here $(I \sim$ $10^{18}-10^{19} \mathrm{~W} / \mathrm{cm}^{2}$ ) high energy protons, mainly originating from contaminant layers of water vapor and hydrocarbons on the target surface [6], are accelerated predominantly from the back of the foil $[12,13]$ and are emitted in the forward direction. According to the target normal sheath acceleration (TNSA) model, a fraction of the laser energy is first converted into relativistic electrons at the front surface of the target. The electrons then propagate through the target and form a dense electron plasma sheath at the rear surface $[14,15]$. The electric field associated with the plasma sheath, which can be of the order of a few $\mathrm{TV} / \mathrm{m}$, ionizes the back of the target and rapidly accelerates ions normal to the initially unperturbed surface. After this initial phase the ions stream into vacuum together with the electrons, which form a Debye sheath at the front of the ion beam. The charge separation in the Debye sheath results in a peak of the accelerating electric field at the ion front (see [16-18], and references therein). As the beam expands, the fast electrons progressively transfer their energy to the ions, and the accelerating charge separation field decreases until the acceleration ceases.

In this Letter we present the first direct experimental measurement of the electric fields responsible for driving the acceleration of high energy protons from a thin foil irradiated by an intense and short laser pulse. The measurement was performed employing an auxiliary laser accelerated proton beam as a transverse charged particle probe $[7,8]$. The spatial structure and time evolution of the observed accelerating fields support the rear-surface acceleration model. Most notably the electric field due to the initial electron sheath and the predicted peak of the accelerating field at the expanding ion front are detected. The experimental results are in good agreement with fluid and particle-in-cell (PIC) simulations of a thin plasma expansion into a vacuum. A detailed comparison between the experiment and the simulations was performed with the use of a charged particle tracer, which simulates the propagation of the probe proton beam through a given evolving field structure.

The experiment was carried out employing the LULI 100 TW system operating in the chirped pulse amplification mode (CPA). After stretching and amplification the laser pulse was split into two separate pulses $\left(\mathrm{CPA}_{1}\right.$ and $\mathrm{CPA}_{2}$ ), which were separately recompressed to temporal durations of $1.5 \mathrm{ps}$ and $300 \mathrm{fs}$, respectively. $\mathrm{CPA}_{1}$ was focused onto 10 to $40 \mu \mathrm{m}$ thick aluminum and gold foils (interaction target) at an intensity of $3.5 \times 10^{18} \mathrm{~W} / \mathrm{cm}^{2}$ and $\mathrm{CPA}_{2}$ was focused onto a $10 \mu \mathrm{m}$ thick gold foil (proton target) at an intensity of $2 \times 10^{19} \mathrm{~W} / \mathrm{cm}^{2}$, leading to the acceleration of a proton beam from each target. The proton beam from the proton target was employed as a transverse charged particle probe for the accelerating electric fields at the back of the interaction target, as shown in Fig. 1(a). We estimated that the cloud of electrons globally neutralizing the probe proton beam was unable to shield the detected fields at the back of the interaction target, as the local Debye length of the probe beam when reaching the target was much larger than the fields' spatial scale 

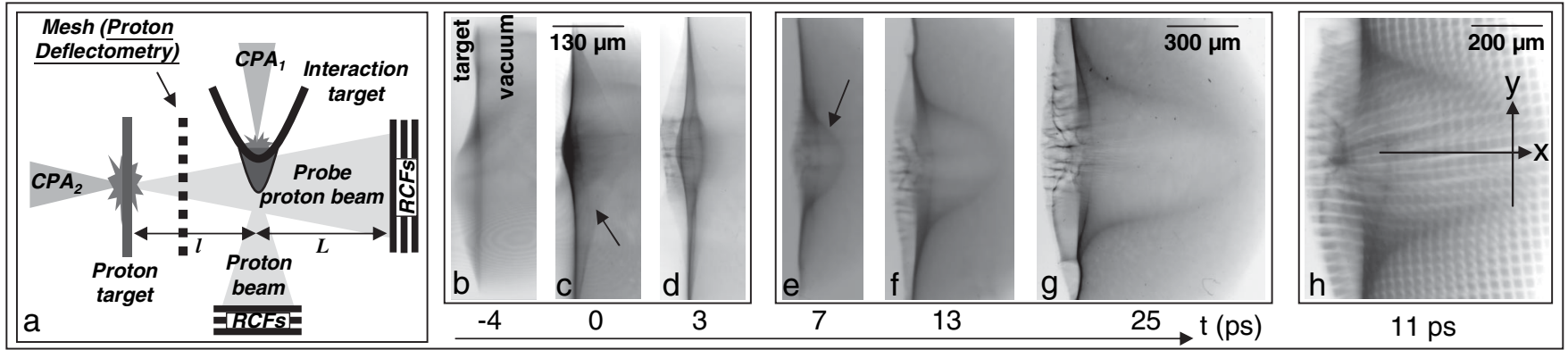

FIG. 1. (a) Experimental setup. (b)-(g) Typical proton imaging data at probing times $\sim-4,0,3,7,13$, and 25 ps, respectively, and (h) proton deflectometry data. The magnification was 30 in (b)-(d) and 15 in (e)-(h). The scales refer to the interaction target plane (scale on detector is $M$ times the scale given in the figure).

length. The interaction targets were bent in order to minimize the deflections due to global target charge-up [7], which would have prevented sampling of the accelerating fields. The time delay between $\mathrm{CPA}_{1}$ and $\mathrm{CPA}_{2}$, and therefore the proton probing time, could be optically adjusted with picosecond precision.

Proton probing techniques exploit the fact that the proton source, while being physically extended, is highly laminar and hence equivalent to a virtual point source [4,5]. In proton imaging, a point projection of the probed region is obtained $[7,8]$ with a spatial resolution set by the virtual source size, which is typically of the order of a few $\mu \mathrm{m}[4,5]$, and a magnification given by $M \simeq(L+l) / l$ [see Fig. 1(a)]. In the experiment $l$ was either 1 or $2 \mathrm{~mm}$, while $L$ was $\sim 30 \mathrm{~mm}$, giving $M$ equal to either $\sim 30$ or $\sim 15$. This technique is mainly sensitive to field gradients, which are detected via proton density modulations in the probe beam cross section. In proton deflectometry [8] a mesh is additionally inserted between the proton target and the interaction target in order to preimprint a periodical pattern on the proton beam cross section. Proton deflections can be measured from the mesh imprint deformation, providing a direct measurement of the fields. The mesh employed in the experiment was a $16.5 \mu \mathrm{m}$ pitch, $5 \mu \mathrm{m}$ bar width, $7 \mu \mathrm{m}$ thick $\mathrm{Cu}$ grid placed at $1 \mathrm{~mm}$ from the proton target (with $l=2 \mathrm{~mm}$ ). The proton beams emitted from the two targets were detected employing stacks of radiochromic films (RCFs) [19]. The multilayer arrangement of the detector provided a spectral and temporal multiframe capability [7], with a temporal resolution in the range 1$10 \mathrm{ps}$, as set by the finite transit time of the protons through the observed field structures.

In Figs. 1(b)-1(g) typical proton imaging data are shown. In the images darker regions correspond to higher proton densities in the probe beam cross section. Each frame corresponds to a different probing time. Two qualitatively different structures can be seen developing in time. Around the peak of the interaction of $\mathrm{CPA}_{1}$ with the bent foil $(t=0)$ a transient, pronounced deflection of the probe protons is observed [Fig. 1(c)]. The probing protons are deflected away from the interaction target rear surface and are redistributed over a bell-shaped extended region [dark region indicated by arrow in Fig. 1(c)]. This deflection vanishes after a few ps, as can be seen by comparing Figs. 1(c) and 1(d). At later times a front expanding from the back of the interaction target is observed. The front is delimited by a bell-shaped thin region of proton accumulation [dark region indicated by the arrow in Fig. 1(e)], which becomes fainter as time goes on. Behind the front the probe proton density is not significantly perturbed.

We interpret these data in the framework of the TNSA model [14-18]. The initial transient deflection can be attributed to the predicted strong electric field associated with the initial dense and hot electron sheath. Under the assumption of small angular deflections, an estimate of the peak electric field can be obtained from the formula $\langle E\rangle \simeq$ $2 \mathcal{E}_{p} \delta x / e L b$, where $\mathcal{E}_{p}$ is the proton energy, $\delta x$ is the proton displacement in the detector plane, and $b$ is the length of the path traveled by the protons through the field. The maximum deflection is experienced by the protons which are displaced from the interaction target rear surface to the apex of the bell, that is, referring to Fig. 1(c), $\delta x \sim$ $4 \mathrm{~mm}$. Taking into account that $\mathcal{E}_{p} \sim 10 \mathrm{MeV}, \quad b \sim$ $100 \mu \mathrm{m}$ (as roughly given by the curvature of the target), and $L=29 \mathrm{~mm}$, the peak electric field on axis can be estimated as $\sim 3 \times 10^{10} \mathrm{~V} / \mathrm{m}$. Note that this value is spatially and temporally averaged over the proton path, implying that the maximum field is much larger, as predicted by numerical simulations of thin plasma expansion into a vacuum (see below). In the same framework the pattern observed at later times [Figs. $1(\mathrm{e})-1(\mathrm{~g})$ ] relates to the predicted expanding proton front [16-18]. The piling up of the probe protons is due to the sudden drop of the electric field ahead of the front. The fact that the dark line delimiting the front becomes less marked at later time is consistent with the expected increase of the field gradient scale length. Finally, the fact that the probe proton density behind the front is not significantly perturbed suggests the absence of strong field gradients in this region [16-18].

These results were confirmed and set in a more quantitative form with the use of proton deflectometry measure- 
ments. In proton deflectograms [Fig. 1(h)] the bell-shaped expanding front observed at late probing times is marked by a clear and sudden shift of the mesh imprint lines. Note, in particular, that the vertical lines remain nearly straight behind the front [Fig. 1(h)] and that the shift along the $x$ axis indicated in Fig. 1(h) has a peak in correspondence with the front position [Fig. 2(c), scatter graph]. This deflection pattern reveals an electric field which peaks at the front and is uniform and substantially smaller behind it. The electric field corresponding to the peak in the deflection can be estimated by considering that the maximum line shift along the $x$ axis indicated in Fig. 1(h) is $\delta x \sim$ $1 \mathrm{~mm}$ [Fig. 2(c)], where $b \sim 500 \mu \mathrm{m}$ [as given, under the assumption of cylindrical symmetry, by the width $\Delta y$ of the region where the deflection is non-negligible; see Fig. 2(d)]. In this case $L=28 \mathrm{~mm}$ and $\mathcal{E}_{p} \sim 7 \mathrm{MeV}$, leading to $\langle E\rangle \simeq 2 \mathcal{E}_{p} \delta x / e L b \simeq 10^{9} \mathrm{~V} / \mathrm{m}$. Again this value is spatially and temporally averaged over the probe proton path, implying that the maximum field should be larger (see below).

The experimentally measured velocity of the expanding front [Figs. $1(\mathrm{e})-1(\mathrm{~g})]$ is $3-4 \times 10^{7} \mathrm{~m} / \mathrm{s}$. This is consistent with the detected high energy spectral cutoff of $\sim 6-7 \mathrm{MeV}$ of the proton beam emitted from the interaction target, taking also into account that, due to the RCF detection threshold, the measure slightly underestimates the cutoff. The expansion velocity and spectral cutoff decrease consistently when the thickness of the interaction target increases, as expected since for thicker targets the spread of the hot electrons within the target will increase,

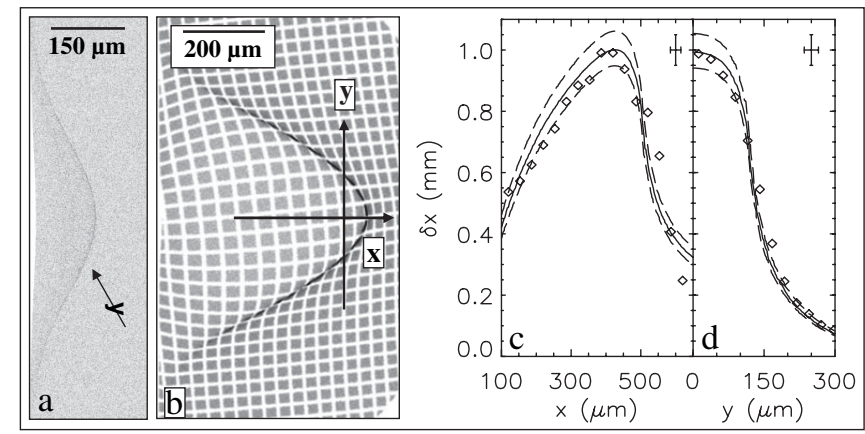

FIG. 2. (a) Particle tracing of the initial transient field. (b) Particle tracing of the expanding front. The scales refer to the interaction target plane. (c) Comparison between the shift of the vertical mesh lines along the $x$ axis indicated in Fig. 1(h) as measured in the detector plane (scatter graph) and the proton deflections in the $x$ direction along the $x$ axis indicated in (b) as obtained from particle tracing simulations (line graphs). The deflections and line shifts are plotted vs the unperturbed proton position in the interaction plane. The solid line corresponds to the mean proton energy detected by the RCF layer in the experiment. The dashed lines correspond to the minimum and maximum proton energies detected by the RCF. (d) Same as in (c), but along the $y$ axes indicated in (b) and in Fig. 1(h), respectively. resulting in a reduced sheath electron density and therefore in a smaller accelerating field. The front velocity at different emission angles is consistent with a laminar flow. The front gains $\sim 70 \%$ of the final velocity in the first 2 ps after the interaction, due to the field associated with the initial electron sheath. Only $\sim 30 \%$ of the final velocity is acquired at later times in the expansion process.

We compared the experimental results with simulations of collisionless thin plasma expansion into a vacuum. The simulations were carried out with the 1D relativistic and electrostatic PIC code described in [17] and the 1D fluid code described in [18]. The codes take into account that the amount of energy available in the thin foil is finite, leading to a well defined cutoff in the ion energy spectrum. The hydrogenlike ions $\left(m_{i}=1836 m_{e}, Z=1\right)$ were assumed initially cold and at rest. The initial unperturbed electron density $n_{e 0} \sim 3 \times 10^{19} \mathrm{~cm}^{-3}$ was estimated from energy conservation, by assuming a conversion efficiency from laser energy into hot electrons of 10\% [20] and an angular spread of the hot electrons within the target of $20^{\circ}$. In the PIC simulation the initial condition was $n_{i}(x, t=0)=$ $n_{e}(x, t=0)=n_{e 0} \quad$ for $\quad|x|<a / 2 \quad$ and $\quad n_{i}(x, t=0)=$ $n_{e}(x, t=0)=0$ for $|x|>a / 2$, where $a=40 \mu \mathrm{m}$ is the foil thickness. In the fluid simulation the initial ion density was $n_{i}(x, t=0)=n_{e 0}$ for $|x|<a / 2$ and $n_{i}(x, t=0)=0$ for $|x|>a / 2$, while the electron density $n_{e}(x, t=0)$ was calculated, with the condition $n_{e}(x=0, t=0)=n_{e 0}$, under the assumption that the electrons are in equilibrium with the electrostatic potential. The initial electron temperature $T_{e 0} \sim 500 \mathrm{keV}$ was estimated from the electron ponderomotive energy in the laser field [14]. A Maxwellian electron velocity distribution with a single electron temperature, corresponding to the hot electron component, was assumed. We verified that the effect of taking into account two electron temperatures is negligible in our case, as the acceleration process is dominated by the hot electrons. With these prescriptions the simulated expanding front dynamics and the cutoff in the ion energy spectrum resulted in very good agreement with the experiment. Figure 3 shows the electric field profile from the simulations at different times. Differences between the two simulations mainly arise from the fact that, as observed in the PIC simulation and differently from what is assumed in the fluid code, at late times the electron distribution function is not Maxwellian anymore. The initial field, due to the electrons initially escaping from the target, peaks at the foil surface and quickly decreases away from the foil. In the expansion phase the field peak decreases and moves together with the ion front, where a Debye sheath forms. Behind the peak a region of nearly constant field is observed. At $t \sim 0$ the peak field values from the simulations are $\sim 4.5 \times 10^{11} \mathrm{~V} / \mathrm{m}$. At $t \sim 11 \mathrm{ps}$ the fields at peak are $6 \times 10^{9} \mathrm{~V} / \mathrm{m}$ (PIC) and $3 \times 10^{9} \mathrm{~V} / \mathrm{m}$ (fluid), while the field in the plateau region is roughly an order of magnitude smaller. These values are in fair agreement with estimates previously given. 


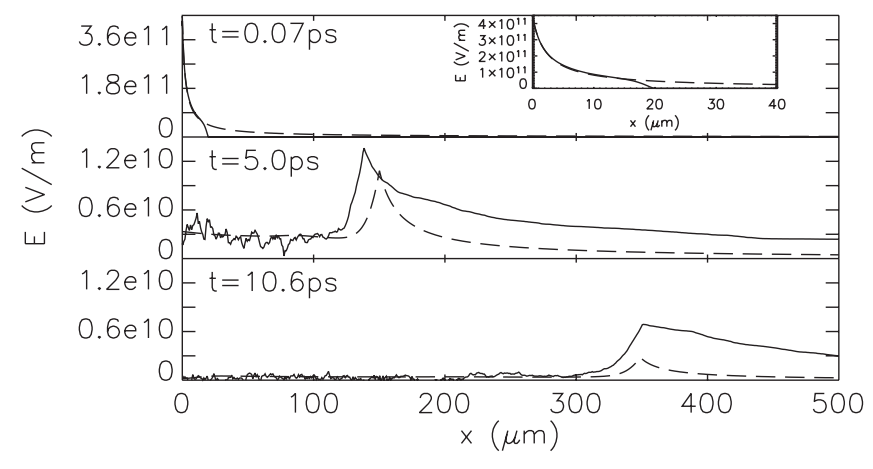

FIG. 3. Field profiles from PIC (solid line) and fluid (dashed line) simulations at three different times and for $T_{e 0}=500 \mathrm{keV}$ and $n_{e 0}=3 \times 10^{19} \mathrm{~cm}^{-3}$.

The proton deflections in given time-dependent electric field configurations were calculated with a 3D charged particle tracer [21]. Both imaging and deflectometry data were simulated. The broad spectral content of the probing proton beam was taken into account. Field patterns with cylindrical symmetry were assumed. The spatial dependence along each field line and the temporal evolution of the electric field were chosen to reproduce the main features observed in the PIC and fluid simulations.

We first simulated the deflection in the transient field corresponding to the case of Fig. 1(c). The field lines were assumed normal to the target surface, with the field peaked at the target surface and decreasing with increasing distance from the target and in time as $\left(1+x / l_{s}\right)^{-1} \times$ $\left(1+t / \tau_{s}\right)^{-1}$ (where $l_{s}$ and $\tau_{s}$ are the field scale length and decay time) as qualitatively suggested by the PIC and fluid simulations. The experimental data could be best reproduced by assuming a field which is null at distances from the target larger than $h$, with $h \sim 20 \mu \mathrm{m}$ giving the best match. This seems to indicate that the electron cloud, and hence the electric field, has a finite extension, as also suggested in [22]. The result from the particle tracing is shown in Fig. 2(a). The best match with the experiment was found for a peak field of $\sim 4-5 \times 10^{11} \mathrm{~V} / \mathrm{m}$, in good agreement with the values from PIC and fluid simulations and with the estimates previously given.

We then simulated the field associated with the expanding ion front, corresponding to the case of Figs. 1(e)-1(h). We assumed that the field lines were straight, originating from a point located behind the target on the symmetry axis, as suggested by the laminarity of the proton source $[4,5]$. Along each field line the field profile reproduced the field structure observed at late times in the PIC and fluid simulations (that is, a plateau region, followed by an exponential rise up to the front field peak and a decay as $(1+$ $\left.x / l_{s}\right)^{-1}$ ahead of the front; see Fig. 3), with the field peak located at an expanding Gaussian-shaped front. The field was assumed to decay in time as $\left(1+t / \tau_{s}\right)^{-1}$ at the peak and as $\left(1+t / \tau_{s}\right)^{-2}$ in the plateau region, as qualitatively suggested by the simulations. In Fig. 2(b) the simulation of the deflectometry shot of Fig. 1(h) is shown. In Figs. 2(c) and 2(d) a direct comparison between the mesh line displacement in the experiment and the proton linear deflection observed in the simulation along the $x$ and $y$ axes indicated in Figs. 1(h) and 2(b) is also shown. The best match with the experiment at $t \sim 11 \mathrm{ps}$ was found for a field of $\sim 2-3 \times 10^{9} \mathrm{~V} / \mathrm{m}$ at peak and of $\sim 10^{8} \mathrm{~V} / \mathrm{m}$ in the plateau region, in good agreement with the values from PIC and fluid simulations and with the estimates previously given. It is important to notice that the experiment could only be reproduced with a field which peaks at the expanding front.

The authors acknowledge the support of the EU program HPRI CT 1999-0052, of Grant No. E1127 from Region Ilede-France, UNR Grant No. DE-FC52-01NV14050, and a Royal Society study visit grant, of DAAD, of British Council-MURST-CRUI, COST and INTAS Networks, and of the SFB/TR18 program. Part of the PIC simulations were performed on the Linux cluster at the CINECA facility (Bologna, Italy), with the support of the INFM supercomputing initiative. The authors acknowledge F. Cornolti, F. Pegoraro, S. V. Bulanov, and M. Cuneo for useful discussions, and the precious assistance of the LULI laser staff.

*Electronic address: m.borghesi@qub.ac.uk

[1] E. L. Clark et al., Phys. Rev. Lett. 84, 670 (2000).

[2] A. Maksimchuk et al., Phys. Rev. Lett. 84, 4108 (2000).

[3] R. A. Snavely et al., Phys. Rev. Lett. 85, 2945 (2000).

[4] M. Borghesi et al., Phys. Rev. Lett. 92, 055003 (2004).

[5] T. E. Cowan et al., Phys. Rev. Lett. 92, 204801 (2004).

[6] S. J. Gitomer et al., Phys. Fluids 29, 2679 (1986).

[7] M. Borghesi et al., Appl. Phys. Lett. 82, 1529 (2003).

[8] A. J. Mackinnon, Rev. Sci. Instrum. 75, 3531 (2004).

[9] M. Santala et al., Appl. Phys. Lett. 78, 19 (2001).

[10] P. Patel et al., Phys. Rev. Lett. 91, 125004 (2003).

[11] M. Roth et al., Phys. Rev. Lett. 86, 436 (2001).

[12] J. Fuchs et al., Phys. Rev. Lett. 94, 045004 (2005).

[13] M. Allen et al., Phys. Rev. Lett. 93, 265004 (2004).

[14] S. Wilks et al., Phys. Plasmas 8, 542 (2001).

[15] S. P. Hatchett et al., Phys. Plasmas 7, 2076 (2000).

[16] P. Mora, Phys. Rev. Lett. 90, 185002 (2003).

[17] S. Betti et al., Plasma Phys. Controlled Fusion 47, 521 (2005).

[18] P. Mora, Phys. Rev. E (to be published).

[19] N. V. Klassen et al., Med. Phys. 24, 1924 (1997).

[20] M. Key et al., Phys. Plasmas 5, 1966 (1998).

[21] A. Schiavi, Ph.D. thesis, Imperial College of Science, Technology and Medicine, London, U.K., 2003.

[22] M. Passoni et al., Laser Part. Beams 22, 163 (2004). 